\title{
Implications of the updated Lung CT Screening Reporting and Data System (Lung-RADS version 1.1) for lung cancer screening
}

\author{
Spencer C. Dyer, Brian J. Bartholmai, Chi Wan Koo \\ Department of Radiology, Mayo Clinic, Rochester, MN, USA \\ Contributions: (I) Conception and design: SC Dyer, CW Ko; (II) Administrative support: SC Dyer; (III) Provision of study materials or patients: \\ None; (IV) Collection and assembly of data: All authors; (V) Data analysis and interpretation: All authors; (VI) Manuscript writing: All authors; (VII) \\ Final approval of manuscript: All authors. \\ Correspondence to: Spencer Dyer. 200 First Street SW, Rochester, MN 55905, USA. Email: dyer.spencer@mayo.edu.
}

\begin{abstract}
Lung cancer remains the leading cause of cancer death in the United States. Screening with low-dose computed tomography (LDCT) has been proven to aid in early detection of lung cancer and reduce disease specific mortality. In 2014, the American College of Radiology (ACR) released version 1.0 of the Lung CT Screening Reporting and Data System (Lung-RADS) as a quality tool to standardize the reporting of lung cancer screening LDCT. In 2019, 5 years after the implementation of Lung-RADS version 1.0 the ACR released the updated Lung-RADS version 1.1 which incorporates initial experience with lung cancer screening. In this review, we outline the implications of the changes and additions in Lung-RADS version 1.1 and examine relevant literature for many of the updates. We also highlight several challenges and opportunities as Lung-RADS version 1.1 is implemented in lung cancer screening programs.
\end{abstract}

Keywords: Lung cancer; cancer screening; pulmonary nodule, multiple; computed tomography, volumetric

Submitted Mar 16, 2020. Accepted for publication Jun 29, 2020.

doi: $10.21037 /$ jtd-2019-cptn-02

View this article at: http://dx.doi.org/10.21037/jtd-2019-cptn-02

\section{Introduction}

Lung cancer remains the leading cause of cancer related deaths in the United States, estimated to account for 142,670 deaths in 2019 (1). In the National Lung Screening Trial (2), lung cancer screening with low dose computed tomography (LDCT) was found to aid in early detection of and reduced mortality due to lung cancer (2). One concern in screening a population for lung cancer is the high rate of false positive tests (3). In 2014, based on the results of several screening trials, the American College of Radiology (ACR) released version 1.0 of the Lung CT Screening Reporting and Data System (Lung-RADS) (4). This is a standardized method of reporting with recommendations for management of pulmonary nodules detected on lung cancer screening CT, analogous to the Breast ImagingReporting and Data System framework for breast cancer screening and reporting (5). When utilized, it can reduce the false positive rate in lung cancer screening without increasing the rates of false negative results $(3,6)$. LungRADS is now deeply embedded as a quality metric on which regulation and reimbursement is determined by the Centers for Medicare and Medicaid (7). During the first 5 years of nationwide lung cancer screening, there has been a significant accumulation of data and experience with many opportunities for continued learning.

In 2019 the ACR released the updated version 1.1, of Lung-RADS given new knowledge gained (8). While many similarities exist between versions, multiple changes found in version 1.1 were made with the intent to adapt to new evidence in the field and to render the system more appropriate for the current lung cancer screening environment. The ACR provides an excellent resource outlining specific modifications implemented in version 1.1 (9). These changes are summarized in Table 1. In this review we discuss relevant literature for many of these updates, as well as some of the implications for future screening programs that carry out these changes. 
Table 1 Updates to version 1.1 of Lung-RADS

\begin{tabular}{lll}
\hline Categories & Additions & Revisions \\
\hline 0 & NA & NA \\
1 & NA & NA \\
2 & Perifissural nodule $(\mathrm{s})<10 \mathrm{~mm}$ & Nonsolid nodules $<30 \mathrm{~mm}$ OR $\geq 30 \mathrm{~mm}$ in unchanged/slowly \\
& growing nodule $(<1.5 \mathrm{~mm})$
\end{tabular}

NA, not applicable.

\section{Revisions}

\section{Nonsolid/ground-glass nodules}

In the Lung-RADS 1.1 update, the measurement thresholds for nonsolid nodules have been revised for categories 2 and 3 (8). In Lung-RADS 1.0, the guidelines defined a nonsolid nodule as a category 2 if it measured $<20 \mathrm{~mm}$ at baseline, or $\geq 20 \mathrm{~mm}$ if unchanged or slowly growing (4). In the update, nonsolid nodules are considered to be category 2 if they measure $<30 \mathrm{~mm}$ at baseline, or $\geq 30 \mathrm{~mm}$ if unchanged or slowly growing. Similarly, in the 1.0 version, a nonsolid nodule measuring $\geq 20 \mathrm{~mm}$ on a baseline CT, or a new nodule, was considered as category 3 ; in the 1.1 update the measurement threshold has been increased to $\geq 30 \mathrm{~mm}$ at baseline, or new on subsequent screening.

To our knowledge there are no studies specifically investigating a threshold of $30 \mathrm{~mm}$ in determining the follow-up course for nonsolid/ground glass nodules. There are, however, multiple studies characterizing the natural course of ground glass nodules (GGNs) (10-13). A patient cohort without a history of malignancy that included 89 pure ground-glass nodules (PGGNs) was followed for a median of 59 months (10). Of these 89 PGGNs, 76 did not change in size, one decreased in size, and 12 increased in size. Of the 12 patients with PGGNs of increasing size, 11 underwent surgical resection and were found to have primary lung adenocarcinoma. Size was found to be a significant risk factor for subsequent growth with $50 \%$ of the GGNs in the growth category initially measuring $>8 \mathrm{~mm}$.
In another group, patients found to have nonsolid and partsolid nodules were followed for up to 136 months, with a mean follow-up of 29 months (12). The investigators found that larger nodules were more likely to grow for both nonsolid and part-solid types. Kakinuma et al. also confirmed that the initial size of PGGNs is the primary predictive factor in determining which nodules will grow (11). Pure GGNs larger than $10 \mathrm{~mm}$ were significantly more likely to demonstrate growth of $\geq 2 \mathrm{~mm}$ in follow-up exams (Figure 1).

Yoon et al. studied a group of 338 patients with 689 GGNs (14). Over a median follow-up of 21.8 months, 79 of the GGNs in 55 patients grew. Within the growth group, nodule size $\geq 10 \mathrm{~mm}$ was again found to be a risk factor for future growth. Additionally, a patient history of prior malignancy and the presence of a single GGN were found to be independent risk factors for future growth, whereas the presence of a solid portion did not correlate with growth risk in their study. They hypothesized that this may be due to resection of part-solid nodules without observation due to their malignant potential (14). Not only is a part-solid component known to confer a greater risk of malignancy, increasing attenuation of GGNs has been shown to be an independent risk factor for future growth and invasiveness $(15,16)$.

Tang and colleagues added another dimension to the analysis of GGNs by considering true growth (growth $\geq 2 \mathrm{~mm}$ ), substantial growth (growth $\geq 5 \mathrm{~mm}$ ), and stage shift (a shift in TNM classification and staging system) (13). Of 93 

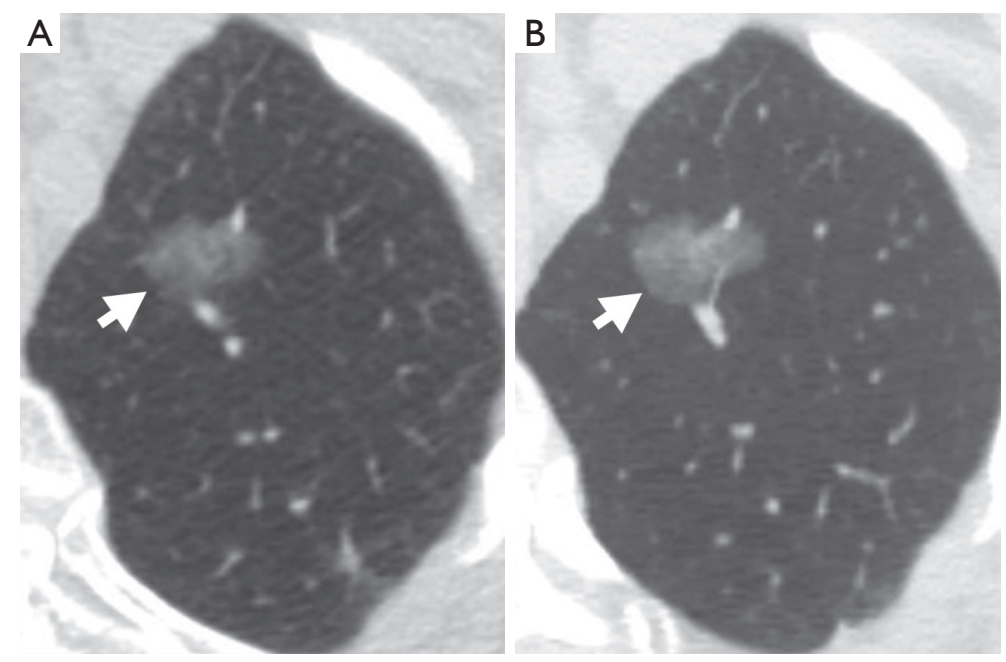

Figure 1 Growth of a large pure ground glass nodule (PGGN). Axial computed tomography images show a 14 mm left apical PGGN (arrows) (A) that demonstrated growth of $2 \mathrm{~mm}$ at 9-month follow-up (B).

patients with a dominant PGGN, 37 had true growth, 17 had substantial growth, and 4 had stage shift. The mean time for true growth was 6.95 years, the mean time for substantial growth was 9.43 years, and the mean time for stage shift was 12.17 years. Of the 93 patients, 36 underwent surgical excision. All of these GGNs were demonstrated to be neoplastic, with 25 classified as invasive adenocarcinoma.

Although the overall evidence is suggestive that PGGNs follow an indolent course, a significant proportion may eventually prove to be malignant and, therefore, warrant surveillance. Based on data from 44 resected PGGNs, a logistic regression model estimated the risk of invasive adenocarcinoma to be $8.1 \%$ for a PGGN with a maximal diameter of $5 \mathrm{~mm}$, and to be $71.6 \%$ with a maximal diameter of $20 \mathrm{~mm}$ (17). Indeed, new nonsolid nodules on lung screening LDCT are more likely to be malignant than solid nodules (18). The management of new GGNs found on follow-up screening has been studied both in the Early Lung Cancer Action Project and in the NELSON participants $(19,20)$. The incidence of new nonsolid nodules in the screening population was low, and most of these nodules resolved on follow-up. Although the number of subjects was low, two out of four persistent nonsolid nodules were found to be premalignant (20).

Despite the malignant potential of PGGNs, Gulati et al. found that none of the 16 patients who declined treatment of suspicious GGNs after biopsy had adverse events related to delaying treatment (21). In addition, none of the 16 died from lung cancer within the 10-year study period. Four did eventually undergo surgical resection; however, none had evidence of metastatic disease.

Based on current evidence, it is likely that the LungRADS framework underestimates the probability of malignancy in GGNs given their inclusion in categories 2 and 3 , which estimate a $<1 \%$ and $1-2 \%$ risk of malignancy, respectively $(18,22)$. However, as has been demonstrated, the natural progression of PGGNs is usually protracted; there is typically little risk to the patient to continue annual follow-up $(21,23)$. In particular, the evidence suggests that any GGN demonstrated at baseline or on a new exam measuring $>10 \mathrm{~mm}$ has a high likelihood of further growth $(11,14,17)$. Risk factors such as prior malignancy, increased age, or large initial size of the nodule are likely predictors of progression of these nodules $(12,14)$. Long term longitudinal studies will be informative in assessing the ultimate effects of this update.

\section{Nodule measurement}

In the Lung-RADS 1.1 framework, the definition of nodule growth as $>1.5 \mathrm{~mm}$ based on the average of linear twodimensional measurements remains. However, nodule measurement underwent two notable changes. First, a volumetric measurement is now included in addition to two-dimensional measurements. Second, regarding twodimensional measurements, the guidelines now recommend measuring to one decimal point and reporting the average diameter to one decimal point. On the other hand, the 
Fleischner Society warned that measurements to $0.1 \mathrm{~mm}$ were likely imprecise for pulmonary nodules (24). Indeed, they suggest that nodules smaller than $3 \mathrm{~mm}$ are too small to accurately measure. To our knowledge, no studies have assessed the utility of measurement to the first decimal. As the Lung-RADS 1.1 framework is adopted, it will be instructive for future studies to evaluate the value of this new requirement.

Although the Fleischner Society noted in 2017 that linear measurement remains the standard of practice (25), linear two-dimensional nodule measurements are problematic from the perspective of consistency and reliability, especially considering most malignant nodules have irregular growth patterns. Revel et al. demonstrated significant intra- and interreader variability in measurement of solid pulmonary nodules to the extent that a change in size of less than $1.73 \mathrm{~mm} \mathrm{had}$ only a $5 \%$ chance of corresponding to real change (26).

Therefore, a notable addition in the Lung-RADS 1.1 update is the inclusion of thresholds for volumetric measurements. While not applied in the NLST trial, volumetric measurements were used in the NELSON trial (27). Benefits of volumetric measurements, such as reproducibility, potential three-dimensional analysis of the entire nodule, and increased sensitivity in detecting nodule growth, have led some to promote volumetric measurement as the preferred method of measuring nodule size and growth determination in lung cancer screening (28-30).

\section{Additions}

\section{Volumetry}

The process of volumetric analysis has been well described (28); however, a brief discussion is worth including. The initial step is accurate segmentation of a pulmonary nodule $(28,31)$. The most common approach employed by current software is a semi-automated process where the radiologist places a "seed" inside a pulmonary nodule, and a region growing algorithm is employed to highlight the nodule (Figure 2). Important features within segmentation software are the ability to distinguish the nodule from background parenchyma, vessels, and the pleural wall. Upon completion of automated segmentation, usually there is an option to manually adjust the segmentation, although this has the potential to introduce variability into the measurements (28).

While most software packages for volumetric analysis use a semi-automated approach, development of computer aided detection (CAD) of nodules with automated segmentation is progressing rapidly. Conventional machine learning and deep learning techniques, such as convolutional neural networks, have allowed CAD to function with up to $95 \%$ sensitivity in the detection of pulmonary nodules (32). CAD tools with both automatic segmentation and volumetric analysis capabilities are available. Indeed, a variety of software solutions with varying abilities exist, both commercially and open source $(33,34)$. While some solutions come as a dedicated lung cancer screening workstation; others can be installed and utilized within a PACS that is already in place. Integration and improvement of these tools with PACS software will be an important step in the process of increasing tool access to radiologists participating in lung cancer screening programs (35). We are not aware of studies analyzing the implementation of these tools within a PACS system; this will be a helpful area of research as $\mathrm{CAD}$ and volumetric tools continue to improve.

At this point, important limitations in the implementation of volumetric measurement for pulmonary nodules persist. One major limitation is continued variability of measurements between vendors and even various iterations of the same software. In 2009, six vendor solutions were tested with highly variable measurements from each (36). Three of these vendors' products were again tested in 2014 with similar variability of measurement. However, a notable finding in the latter study was the relative consistency in the change of volumes across vendors (34). Open source and commercial vendors were studied in a multi-institutional retrospective study which concurred with the aforementioned findings, demonstrating that change in volume is relatively consistent regardless of the software solution employed (33). Given this consistency, nodules can be followed using volumetric measurements when determined by the same software solution. However, care must be taken when interpreting volumetric data generated by different software or different versions of the same software (33,34).

Differences in CT acquisition parameters and segmentation algorithms are also important factors that can lead to variability in volumetric measurement. Slice thickness and other protocol-related factors such as the reconstruction kernel and field of view must remain constant for reliable measurements to be performed $(28,37)$. Although some software packages allow customization of options that change the density threshold for segmentation, standardized parameters must exist between practices in order to maintain homogenous and comparable 


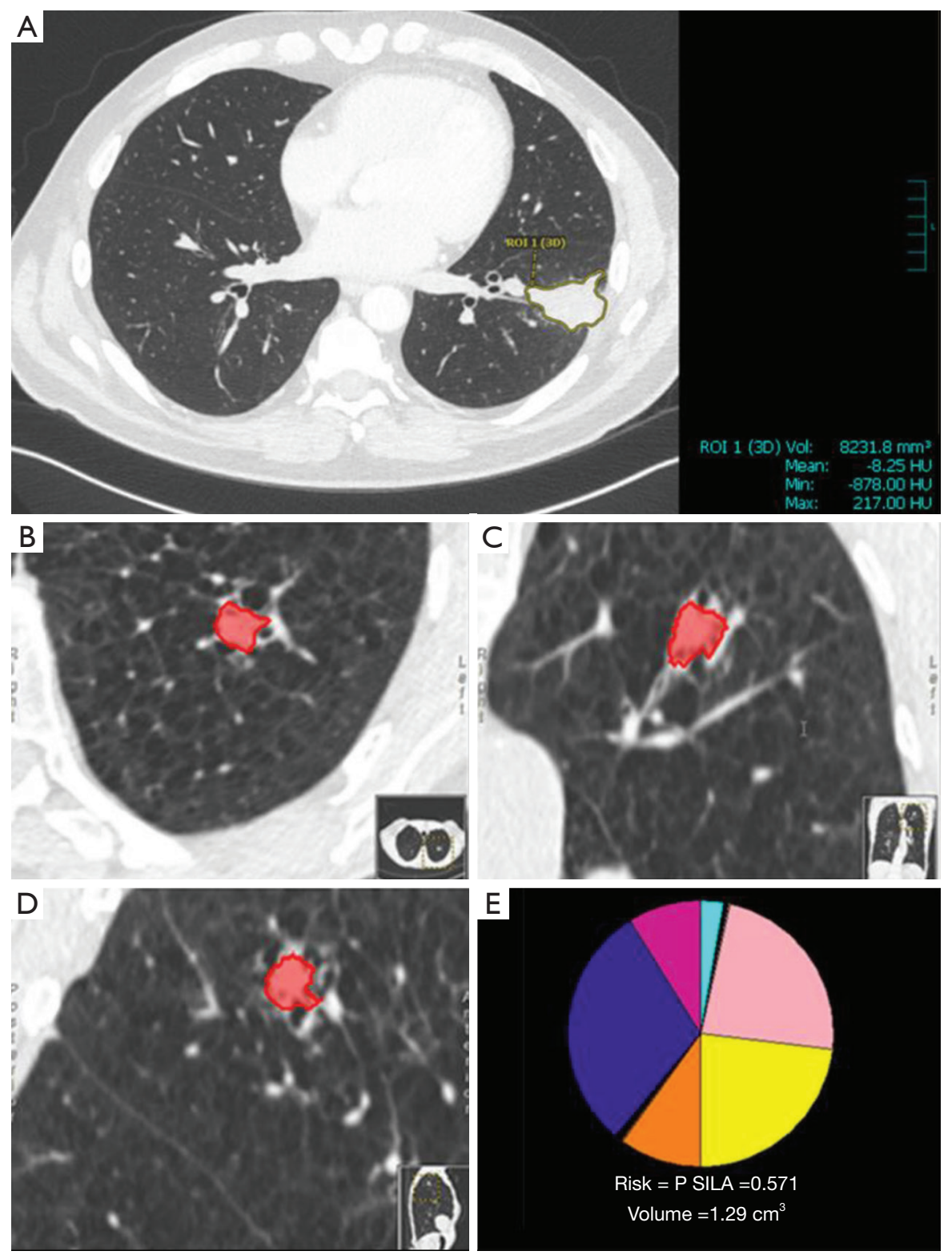

Figure 2 Illustration of pulmonary nodule segmentation and volumetry. (A) Semi-automated segmentation and basic volumetry in a picture archiving and communication system (PACS) integrated program (Visage, Visage Imaging Inc., Richmond, Victoria, Australia). Semiautomated segmentation in axial (B), coronal (C), and sagittal (D) planes of a solid nodule in a different patient (shaded in red), and its resultant volumetric measurement (E) in a standalone image-based risk prediction tool (Computer-Aided Nodule Assessment and Risk Yield, Mayo Clinic, Rochester, MN, USA).

measurements if volumetry is to be widely utilized. Alternatively, advanced, more generalizable algorithms must be developed to compensate for variability of imaging parameters across time and institutions.

Another challenge in nodule volumetric measurement is segmentation of small pulmonary nodules, as current volumetric solutions tend to underestimate the volume of nodules $<5 \mathrm{~mm}(28,38)$. Subsolid nodules provide an additional challenge to segmentation software due to their indistinct borders and relatively similar densities compared to the normal lung parenchyma background. New algorithms are in development with the help of machine 
Table 2 Characteristics of typical and atypical PFNs (12)

\begin{tabular}{|c|c|}
\hline Typical perifissural nodules & Atypical perifissural nodules \\
\hline Solid & Solid \\
\hline Smooth margins & Smooth margins \\
\hline Oval, lentiform, or triangular shape & $\begin{array}{l}\text { Oval, lentiform, triangular shape, or convex on one side and rounded on the other (not influenced } \\
\text { by fissure) }\end{array}$ \\
\hline
\end{tabular}

PFNs, perifissural nodules.

learning to improve nodule segmentation and subtract background details, such as blood vessels and pleura (39). Using a novel technique with CAD and vessel suppression, Lo et al. showed that radiologist sensitivity in detecting suspicious pulmonary nodules can be increased while also decreasing the time of interpretation by $26 \%$ (40). Indeed, deep learning and convolutional neural networks are increasingly able to deliver on tasks such as correct nodule localization and segmentation (32).

Few details have been studied regarding the use of volumetric measurement in clinical practice. One group found that volumetry was helpful in detecting suspicious growth of pulmonary nodules, but altered the decision to biopsy in only a small number of cases (41). On the other hand, the addition of volumetric measurements alone has been shown to increase the ability of several models to predict malignancy (42). As volumetric measurements come into wider use, additional tools will become available, such as CAD and automatic segmentation, to aid the decisionmaking process. As these tools are advanced, their ability to detect and measure pulmonary nodules with increasing accuracy and efficiency will increase, thus becoming a more important aspect of clinical practice. Until that time, many hurdles must be overcome before implementing volumetric measurements in the setting of lung cancer screening.

\section{Perifissural nodules}

A notable update to the Lung-RADS reporting system is the addition of perifissural nodules (PFNs) $<10 \mathrm{~mm}$ as a specific finding within the benign category (8). As defined in the Lung-RADS framework, a PFN is an oval, lentiform, or triangular-shaped solid nodule with smooth margins. Additionally, these nodules are located on or within $10 \mathrm{~mm}$ of a fissure and are most often below the level of the carina $(43,44)$. In one series, a retrospective analysis of 146 patients undergoing lung cancer screening
CT revealed that up to one third of screened patients had PFNs on screening CT (43). These patients were followed out to 7.5 years, and none of the PFNs developed into malignancy. Another study also demonstrated the benignity of PFNs using data from the Dutch-Belgian Randomized Lung Cancer Screening Trial (Dutch acronym: NELSON trial) (45). In the NELSON cohort, 794 of 4,026 detected nodules (19.7\%) represented PFNs. The majority of typical and atypical PFNs detected at baseline remained stable or regressed at first follow-up CT. Of the PFNs that grew, none were found to be malignant, even at 5.5 years of follow-up. An additional analysis of NELSON trial participants investigated patients presenting with new solid nodules at 1,3 , and 5.5 years after baseline (46). In these participants, 1,494 new nodules were detected; of the 91 malignant nodules detected, none were classified as a typical or atypical PFN. Given that these benign PFNs might have artificially increased the false positive rate of lung cancer screening, it would be instructive for future studies to assess whether excluding these PFNs decreases false positive rates.

While prior studies support the classification of PFNs as benign out-of-hand, there can be variability in their evaluation. In a study analyzing cancerous and noncancerous nodules from the NLST database, significant inter-reader variability was demonstrated in classifying small solid nodules accurately as PFNs (44). Both de Hoop (45) and Schreuder (44) distinguished between "typical" and "atypical" PFNs. Typical PFNs were defined as smoothly marginated, small, solid, lentiform/oval/triangular-shaped nodules attached to or within $10 \mathrm{~mm}$ of a fissure, with extending linear densities $(44,45)$ (Table 2, Figure 3). An atypical PFN was described by de Hoop et al. (45) as a nodule otherwise meeting criteria for a PFN that is not visibly attached to a fissure, while Schreuder et al. (44) described an atypical PFN as a nodule meeting two of three major characteristics of a PFN (namely typical shape, attachment to a fissure, and presence of extending linear 

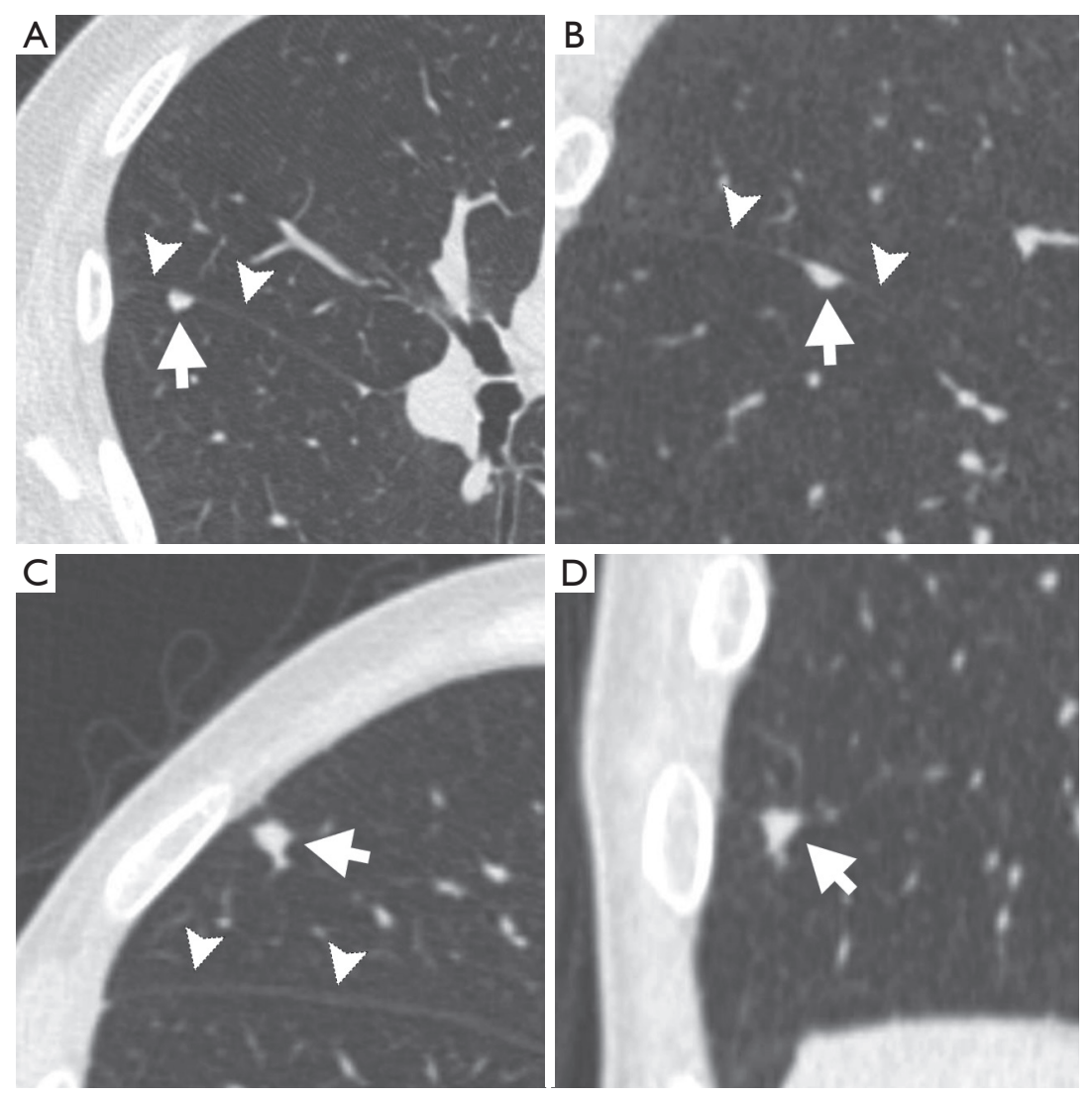

Figure 3 Samples of typical and atypical perifissural nodules on computed tomography. (A) Axial section showing a typical PFN (arrow) attached to the right major fissure (arrowheads). (B) Coronal section with another typical PFN (arrow) attached to the minor fissure (arrowheads). An atypical PFN (arrow) in axial (C) and coronal (D) sections without visible attachment to the right major fissure (arrowheads in C).

densities). None of the nodules defined as atypical from the NELSON database were found to be malignant (45). However, inter-reader variability in classifying typical and atypical PFNs can lead to false negative results. Schreuder et al. found that 13 pulmonary malignancies were misclassified as a PFN by at least one reader (44); 11 of 13 of these were located in the upper lobes, and two were attached to a fissure. Therefore, although studies strongly supported the Lung-RADS 1.1 update to include PFNs $<10 \mathrm{~mm}$ in the benign category, care should be taken when assessing these nodules to accurately identify the features of typical and atypical PFNs in order to avoid the possibility of a false negative finding.

\section{Infectious/inflammatory nodules}

In addition to changes in measurement and classification, the ACR now includes follow-up recommendations for indeterminate nodules within the framework of Lung-
RADS. This change allows radiologists some flexibility when reporting solid nodules on screening CT that qualify as category 4B or 4X lesions. According to LungRADS version 1.1, such nodules may be followed up on a one-month basis to check for resolution of possible inflammatory or infectious etiology (9).

Few studies have detailed the rate of resolution among solid nodules detected on CT. One study analyzed 964 indeterminate solid nodules found in 770 patients screened as part of the NELSON cohort (47). Of the 964 nodules, 97 nodules in 75 participants resolved. Of the nodules that resolved, $75 \%$ did so spontaneously on the 3-month follow-up CT. Non-peripheral location and spiculated borders increased the chances that a nodule would be found to have resolved at follow-up. Additionally, a higher percentage of nodules measuring $\geq 8 \mathrm{~mm}$ at baseline resolved compared to smaller nodules, and at a more rapid rate (47). Notably, solid nodules are far less likely to resolve than GGNs or part solid nodules (PSNs) (48). Yu et al. found within their cohort that only 22\% 

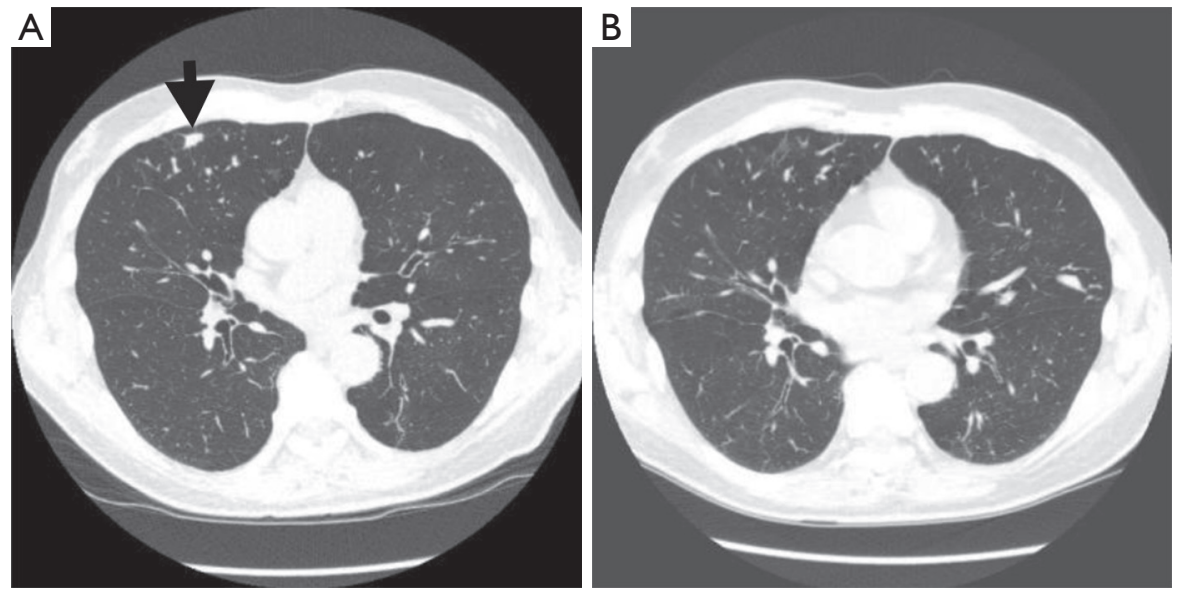

Figure 4 Illustration of a resolving solid nodule. Axial image demonstrates a new spiculated nodule (arrow) detected on screening computed tomography (A) that resolved on a 4-month follow-up CT (B).

of solid nodules resolved during follow-up compared with $67 \%$ of PGGNs and $55 \%$ of mixed GGNs (48). Both Yu's and Lee's groups found that age $\leq 55$ years and blood eosinophilia were predictors for nodule resolution $(48,49)$. A multiplicity of PSNs, a large solid component, and irregular borders were also predictors for resolution (49) (Figure 4).

The time to resolution for GGNs, PSNs, and solid nodules is unfortunately not clear (47-49). Zhao et al. (47) found that of nodules that resolved, 75 of 97 did so by the 3 month follow-up CT, and Lee et al. (49) defined transience as reduction or resolution of PSNs at 3 months. Subjects studied by $\mathrm{Yu}$ et al. had a mean follow-up duration of 4.7 months, although the time to resolution was not specifically described (48). Diederich et al. found that among 133 resolving pulmonary nodules, $80 \%$ completely resolved in a mean of 492 days (50). The majority of these nodules $(85 \%)$ were solid.

The Lung-RADS 1.1 update suggests a one-month follow-up CT for new solid nodules which meet criteria for categories $4 \mathrm{~B}$ or $4 \mathrm{X}$. It is likely that over one month, many transient nodules may at least partially resolve (47), while a shorter follow-up time may potentially lead to a greater number of false positive results, particularly in younger patients $(48,49)$. It would be informative to assess if a one-month follow-up period is the optimal interval for surveillance of these new nodules.

\section{Supplemental resources}

\section{Risk calculators}

In both the 1.0 and 1.1 iterations of Lung-RADS, the ACR provides a link to the Brock University nodule malignancy prediction calculator as an adjunct to assist in decisionmaking for management of pulmonary nodules detected on screening. Although the Brock calculator is unchanged, a discussion of risk calculators does have implications for implementation and continued improvement of a lung cancer screening program.

Developed by McWilliams et al., this calculator is listed as "Nodule Malignancy Prediction Calculator" on the Brock University website (51). The data that needs to be entered into the calculator includes patient age, sex, family history of cancer, presence of emphysema, the type and number of nodules, nodule location, and presence of spiculation. In the literature, this calculator has been referred to as the Vancouver risk calculator and Pan-Canadian Early Detection of Lung Cancer (PanCan) model, but we will refer to this risk calculator hereafter as the Brock calculator. This tool is referenced specifically to facilitate the decision to pursue further diagnostic or functional imaging and/or to proceed with biopsy (8).

Many risk calculators have been developed based on disparate populations with variable lung cancer risk (52). The Brock calculator is particularly useful because it was developed using a lung cancer screening population (52). In presenting the Brock calculator, McWilliams et al. pointed out that while a nodule's size is the most important predictor of malignancy, in a number of individuals the largest lung nodule is not necessarily malignant (51). Sixteen of 102 (15.6\%) cancers within the studied PanCan participants were found in the second largest nodule. This detail highlights the need for risk calculators to aid in 
guiding management to target nodules most likely to be malignant (51).

White et al. recently compared the ACR Lung-RADS with the Brock calculator using data from the NLST cohort (53). On both a per nodule and per patient basis, the Brock calculator was more specific and accurate in detecting malignancy than the Lung-RADS categorization. However, sensitivity was not found to be significantly different. When applied to the NELSON cohort at a single time point, the Brock calculator outperformed Lung-RADS in identifying malignant nodules (54). The Brock calculator has also performed well in cohorts from the Netherlands (55) and United Kingdom (56).

When applied to a community lung cancer screening program, the Brock risk calculator performed well at a population level in identifying patients with at least one pulmonary nodule with a high probability of cancer (57). However, when applied on an individual basis, the risk calculator performed poorly in distinguishing malignant from benign lesions for nodules $>6 \mathrm{~mm}$. Hammer et al. found similar challenges when applying risk calculators to large ( $\geq 8 \mathrm{~mm}$ ) nodules in their population (58). They identified 86 pulmonary nodules, $69 \%$ of which were proven to be malignant by biopsy (39/59) and surgical excision (18/59). However, all tested risk calculators underestimated the risk of malignancy within this subset of large nodules. Specifically, the Brock calculator performed the best, but with only a $53 \%$ negative predictive value and an $81 \%$ positive predictive value in this population (58).

An important factor when applying a risk stratification tool is external validation. The Brock calculator has been studied in multiple population settings, but Winter et al. pointed out that few studies have utilized discrimination and calibration metrics in applying this predictive model to a new population (59). Fundamental differences in nodule characteristics between the PanCan and NLST cohort are important considerations when applying this risk calculator to a generalized population $(59,60)$. Prior to recalibration, applying the Brock risk calculator to the NLST cohort overestimated the probability of cancer. However, with recalibration, calculator performance improved (59).

Further evaluation of the performance of Lung-RADS 1.1 , the Brock, and other risk calculators with appropriate external validation will be helpful (59). A major benefit of the Lung-RADS framework is that it enables immediate PACS-side radiologist categorization of pulmonary nodules (53). It also provides standardization for nodule classification. Additionally, the Lung-RADS categories account for growth, while the Brock calculator does not (54). Radiologists and clinicians will likely benefit most by utilizing clinical judgement, having been informed by these risk calculators, in their decision making (58).

\section{Conclusions}

The 1.1 update of Lung-RADS introduces many changes. While some reflect growing knowledge about the behavior of benign or indolent nodules, other changes aim to allow radiologists to use Lung-RADS more flexibly and effectively. It will be instructive to study the effects of these recent Lung-RADS changes by incorporating PFNs into the benign category, measuring nodules to the nearest decimal point, and using risk stratification tools, such as the Brock calculator, separately and in tandem with LungRADS 1.1 in management decisions. As segmentation tools and volumetric analysis are refined and more advanced quantitative tools integrating AI analysis of the imaging characteristics are implemented in practice, the evidence suggests that our ability to detect change earlier will increase. Additionally, advances in machine learning may further aid our efforts to effectively manage patients with suspicious lung nodules. These potential advancements in lung cancer surveillance make for an exciting time in the realm of thoracic imaging.

\section{Acknowledgments}

The authors wish to thank Desiree Lanzino, PT, PhD, for her editorial assistance.

Funding: None.

\section{Footnote}

Provenance and Peer Review: This article was commissioned by the Guest Editor (Chi Wan Koo) for the series "Contemporary Practice in Thoracic Neoplasm Diagnosis, Evaluation and Treatment" published in Fournal of Thoracic Disease. The article was sent for external peer review organized by the Guest Editor and the editorial office.

Conflicts of Interest: The authors have completed the ICMJE uniform disclosure form (available at: http://dx.doi. org/10.21037/jtd-2019-cptn-02). The series "Contemporary practice in thoracic neoplasm diagnosis, evaluation and treatment" was commissioned by the editorial office without any funding or sponsorship. CWK served as the unpaid 
Guest Editor of the series and serves as an unpaid editorial board member of Fournal of Thoracic Disease from Dec 2018 to Nov 2020. BJB reports personal fees from Promedior, LLC, other feel from Imbio, LLC, outside the submitted work. BJB holds intellectual property rights as an inventor of the CANARY software, but does not have any financial relationships regarding this software and this lung cancer risk assessment software is not directly related to the topic of this manuscript. The authors have no other conflicts of interest to declare.

Ethical Statement: The authors are accountable for all aspects of the work in ensuring that questions related to the accuracy or integrity of any part of the work are appropriately investigated and resolved.

Open Access Statement: This is an Open Access article distributed in accordance with the Creative Commons Attribution-NonCommercial-NoDerivs 4.0 International License (CC BY-NC-ND 4.0), which permits the noncommercial replication and distribution of the article with the strict proviso that no changes or edits are made and the original work is properly cited (including links to both the formal publication through the relevant DOI and the license). See: https://creativecommons.org/licenses/by-nc-nd/4.0/.

\section{References}

1. Siegel RL, Miller KD, Jemal A. Cancer statistics, 2019. CA Cancer J Clin 2019;69:7-34.

2. Aberle DR, Adams AM, Berg CD, et al. Reduced lungcancer mortality with low-dose computed tomographic screening. N Engl J Med 2011;365:395-409.

3. Pinsky PF, Gierada DS, Black W, et al. Performance of Lung-RADS in the National Lung Screening Trial: a retrospective assessment. Ann Intern Med 2015;162:485-91.

4. ACoR. Lung-Screening Reporting and Data System (LungRADS) Version 1.0. 2014. 2014. Available online: https://www.acr.org/-/media/ACR/Files/RADS/LungRADS/LungRADS_AssessmentCategories.pdf

5. Martin MD, Kanne JP, Broderick LS, et al. Lung-RADS: Pushing the Limits. Radiographics 2017;37:1975-93.

6. McKee BJ, Regis SM, McKee AB, et al. Performance of ACR Lung-RADS in a clinical CT lung screening program. J Am Coll Radiol 2016;13:R25-9.

7. Aberle DR. Implementing lung cancer screening: the US experience. Clin Radiol 2017;72:401-6.
8. ACoR. Lung-Screening Reporting and Data System (LungRADS) Version 1.1. 2019. Available online: https:// www.acr.org/-/media/ACR/Files/RADS/Lung-RADS/Lun gRADSAssessmentCategoriesv1-1.pdf?la $=$ en

9. ACoR. ACR Lung-RADS - Update 1.1 2019. 2019. Available online: https://www.acr.org/-/media/ACR/Files/ RADS/Lung-RADS/LungRADS-1-1-updates.pdf?la=en

10. Chang B, Hwang JH, Choi YH, et al. Natural history of pure ground-glass opacity lung nodules detected by lowdose CT scan. Chest 2013;143:172-8.

11. Kakinuma R, Noguchi M, Ashizawa K, et al. Natural History of Pulmonary Subsolid Nodules: A Prospective Multicenter Study. J Thorac Oncol 2016;11:1012-28.

12. Matsuguma H, Mori K, Nakahara R, et al. Characteristics of subsolid pulmonary nodules showing growth during follow-up with CT scanning. Chest 2013;143:436-43.

13. Tang EK, Chen CS, Wu CC, et al. Natural History of Persistent Pulmonary Subsolid Nodules: Long-Term Observation of Different Interval Growth. Heart Lung Circ 2019;28:1747-54.

14. Yoon HY, Bae JY, Kim Y, et al. Risk factors associated with an increase in the size of ground-glass lung nodules on chest computed tomography. Thorac Cancer 2019;10:1544-51.

15. Tamura M, Shimizu Y, Yamamoto T, et al. Predictive value of one-dimensional mean computed tomography value of ground-glass opacity on high-resolution images for the possibility of future change. J Thorac Oncol 2014;9:469-72.

16. Kitazawa S, Saeki Y, Kobayashi N, et al. Threedimensional mean CT attenuation value of pure and partsolid ground-glass lung nodules may predict invasiveness in early adenocarcinoma. Clin Radiol 2019;74:944-9.

17. Lee GD, Park CH, Park HS, et al. Lung Adenocarcinoma Invasiveness Risk in Pure Ground-Glass Opacity Lung Nodules Smaller than $2 \mathrm{~cm}$. Thorac Cardiovasc Surg 2019;67:321-8.

18. Henschke CI, Yankelevitz DF, Mirtcheva R, et al. CT screening for lung cancer: frequency and significance of part-solid and nonsolid nodules. AJR Am J Roentgenol 2002;178:1053-7.

19. Henschke CI, Yip R, Smith JP, et al. CT Screening for Lung Cancer: Part-Solid Nodules in Baseline and Annual Repeat Rounds. AJR Am J Roentgenol 2016;207:1176-84.

20. Walter JE, Heuvelmans MA, Yousaf-Khan U, et al. New Subsolid Pulmonary Nodules in Lung Cancer Screening: The NELSON Trial. J Thorac Oncol 2018;13:1410-4.

21. Gulati CM, Schreiner AM, Libby DM, et al. Outcomes of 
unresected ground-glass nodules with cytology suspicious for adenocarcinoma. J Thorac Oncol 2014;9:685-91.

22. Hammer MM, Palazzo LL, Eckel AL, et al. A Decision Analysis of Follow-up and Treatment Algorithms for Nonsolid Pulmonary Nodules. Radiology 2019;290:506-13.

23. Heuvelmans MA, Walter JE, Oudkerk M. Management of baseline and new sub-solid nodules in CT lung cancer screening. Expert Rev Respir Med 2018;12:1-3.

24. Bankier AA, MacMahon H, Goo JM, et al. Recommendations for Measuring Pulmonary Nodules at CT: A Statement from the Fleischner Society. Radiology 2017;285:584-600.

25. MacMahon H, Naidich DP, Goo JM, et al. Guidelines for Management of Incidental Pulmonary Nodules Detected on CT Images: From the Fleischner Society 2017. Radiology 2017;284:228-43.

26. Revel MP, Bissery A, Bienvenu M, et al. Are twodimensional CT measurements of small noncalcified pulmonary nodules reliable? Radiology 2004;231:453-8.

27. Ru Zhao Y, Xie X, de Koning HJ, et al. NELSON lung cancer screening study. Cancer Imaging 2011;11 Spec No A:S79-84.

28. Devaraj A, van Ginneken B, Nair A, et al. Use of Volumetry for Lung Nodule Management: Theory and Practice. Radiology 2017;284:630-44.

29. Han D, Heuvelmans MA, Vliegenthart R, et al. Influence of lung nodule margin on volume- and diameter-based reader variability in CT lung cancer screening. Br J Radiol 2018;91:20170405.

30. Yankelevitz DF, Reeves AP, Kostis WJ, et al. Small pulmonary nodules: volumetrically determined growth rates based on CT evaluation. Radiology 2000;217:251-6.

31. Kuhnigk JM, Dicken V, Bornemann L, et al. Morphological segmentation and partial volume analysis for volumetry of solid pulmonary lesions in thoracic CT scans. IEEE Trans Med Imaging 2006;25:417-34.

32. Lee SM, Seo JB, Yun J, et al. Deep Learning Applications in Chest Radiography and Computed Tomography: Current State of the Art. J Thorac Imaging 2019;34:75-85.

33. Balagurunathan Y, Beers A, Kalpathy-Cramer J, et al. Semi-automated pulmonary nodule interval segmentation using the NLST data. Med Phys 2018;45:1093-107.

34. Zhao YR, van Ooijen PM, Dorrius MD, et al. Comparison of three software systems for semi-automatic volumetry of pulmonary nodules on baseline and follow-up CT examinations. Acta Radiol 2014;55:691-8.

35. Firmino M, Morais AH, Mendoca RM, et al. Computeraided detection system for lung cancer in computed tomography scans: review and future prospects. Biomed Eng Online 2014;13:41.

36. de Hoop B, Gietema H, van Ginneken B, et al. A comparison of six software packages for evaluation of solid lung nodules using semi-automated volumetry: what is the minimum increase in size to detect growth in repeated CT examinations. Eur Radiol 2009;19:800-8.

37. Petrou M, Quint LE, Nan B, et al. Pulmonary nodule volumetric measurement variability as a function of CT slice thickness and nodule morphology. AJR Am J Roentgenol 2007;188:306-12.

38. Xie X, Willemink MJ, de Jong PA, et al. Small irregular pulmonary nodules in low-dose CT: observer detection sensitivity and volumetry accuracy. AJR Am J Roentgenol 2014;202:W202-9.

39. Mukhopadhyay S. A Segmentation Framework of Pulmonary Nodules in Lung CT Images. J Digit Imaging 2016;29:86-103.

40. Lo SB, Freedman MT, Gillis LB, et al. JOURNAL CLUB: Computer-Aided Detection of Lung Nodules on CT With a Computerized Pulmonary Vessel Suppressed Function. AJR Am J Roentgenol 2018;210:480-8.

41. Korst RJ, Lee BE, Krinsky GA, et al. The utility of automated volumetric growth analysis in a dedicated pulmonary nodule clinic. J Thorac Cardiovasc Surg 2011;142:372-7.

42. Mehta HJ, Ravenel JG, Shaftman SR, et al. The utility of nodule volume in the context of malignancy prediction for small pulmonary nodules. Chest 2014;145:464-72.

43. Ahn MI, Gleeson TG, Chan IH, et al. Perifissural nodules seen at CT screening for lung cancer. Radiology 2010;254:949-56.

44. Schreuder A, van Ginneken B, Scholten ET, et al. Classification of CT Pulmonary Opacities as Perifissural Nodules: Reader Variability. Radiology 2018;288:867-75.

45. de Hoop B, van Ginneken B, Gietema H, et al. Pulmonary perifissural nodules on CT scans: rapid growth is not a predictor of malignancy. Radiology 2012;265:611-6.

46. Heuvelmans M, Van Smoorenburg L, Walter J, et al. Lung Cancer Probability in New Perifissural Nodules Detected in a Lung Cancer Screening Study. J Thorac Oncol 2018;13:S567-S.

47. Zhao YR, Heuvelmans MA, Dorrius MD, et al. Features of resolving and nonresolving indeterminate pulmonary nodules at follow-up CT: the NELSON study. Radiology 2014;270:872-9.

48. Yu JY, Lee B, Ju S, et al. Proportion and characteristics of transient nodules in a retrospective analysis of pulmonary 
nodules. Thorac Cancer 2012;3:224-8.

49. Lee SM, Park CM, Goo JM, et al. Transient part-solid nodules detected at screening thin-section CT for lung cancer: comparison with persistent part-solid nodules. Radiology 2010;255:242-51.

50. Diederich S, Hansen J, Wormanns D. Resolving small pulmonary nodules: CT features. Eur Radiol 2005;15:2064-9.

51. McWilliams A, Tammemagi MC, Mayo JR, et al. Probability of cancer in pulmonary nodules detected on first screening CT. N Engl J Med 2013;369:910-9.

52. Choi HK, Ghobrial M, Mazzone PJ. Models to Estimate the Probability of Malignancy in Patients with Pulmonary Nodules. Ann Am Thorac Soc 2018;15:1117-26.

53. White CS, Dharaiya E, Dalal S, et al. Vancouver Risk Calculator Compared with ACR Lung-RADS in Predicting Malignancy: Analysis of the National Lung Screening Trial. Radiology 2019;291:205-11.

54. van Riel SJ, Ciompi F, Jacobs C, et al. Malignancy risk estimation of screen-detected nodules at baseline CT: comparison of the PanCan model, Lung-RADS and NCCN guidelines. Eur Radiol 2017;27:4019-29.

Cite this article as: Dyer SC, Bartholmai BJ, Koo CW. Implications of the updated Lung CT Screening Reporting and Data System (Lung-RADS version 1.1) for lung cancer screening. J Thorac Dis 2020;12(11):6966-6977. doi: 10.21037/jtd2019-cptn-02
55. Chung K, Mets OM, Gerke PK, et al. Brock malignancy risk calculator for pulmonary nodules: validation outside a lung cancer screening population. Thorax 2018;73:857-63.

56. Al-Ameri A, Malhotra P, Thygesen H, et al. Risk of malignancy in pulmonary nodules: A validation study of four prediction models. Lung Cancer 2015;89:27-30.

57. Gilbert CR, Carlson AS, Wilshire CL, et al. The decision to biopsy in a lung cancer screening program: Potential impact of risk calculators. J Med Screen 2019;26:50-6.

58. Hammer MM, Nachiappan AC, Barbosa EJM Jr. Limited Utility of Pulmonary Nodule Risk Calculators for Managing Large Nodules. Curr Probl Diagn Radiol 2018;47:23-7.

59. Winter A, Aberle DR, Hsu W. External validation and recalibration of the Brock model to predict probability of cancer in pulmonary nodules using NLST data. Thorax 2019;74:551-63.

60. White CS, Dharaiya E, Campbell E, et al. The Vancouver Lung Cancer Risk Prediction Model: Assessment by Using a Subset of the National Lung Screening Trial Cohort. Radiology 2017;283:264-72. 